\title{
Face, portrait, mask - using a parameterised system to explore synthetic face space
}

\author{
Steve DiPaola \\ Simon Fraser University \\ 250 - 13450 102nd Avenue \\ Surrey, BC V3T 0A3, CANADA \\ sdipaola@sfu.ca
}

\begin{abstract}
New technological tools are allowing the authorship of computer generated faces that can easily move between very realistic, to cartoon-like, to painterly or even iconified - both in depiction and movement. These systems are beginning to allow artists, scientists and scholars to explore the notion of 'face space', whether as a realistic emotive character, an artistic portrait or symbolic facial mask, in new ways, that give a deeper understanding of how faces work as an expressive and communicative medium. We overview our computer facial suite of tools, which using a hierarchical parameterisation approach, has been used as a comprehensive frame work in several interdisciplinary, industrial and cognitive science applications.
\end{abstract}

Computer graphics. Facial animation. Parametric spaces. Painterly rendering. Expression systems.

\section{INTRODUCTION}

We overview our interdisciplinary computational tools that build parameterised synthetic facial depiction, expression and research systems. It allows artists, scientists and scholars to move through a space of faces from artistic to realistic. With new computational systems it is possible to conceptually, compose and explore in higher level conceptual spaces - with our system that space is facial emotion and depiction. We are interested in building art and science systems that support exploring these spaces and in particular report on our software-based facial toolkit and resulting experiments using parameter spaces in face based new media.

With new technological artistic tools that allow us to author computer generated and manipulated faces that can be as real or as abstract or as iconified as we choose, what aesthetic and cultural communication language do we elicit? Is it the historically rich language of the fine art portrait the abstracted artefact of the human face? What happens when this portrait animates, conveying lifelike human facial emotion - does it cease to be a portrait and instead moves into the realm of embodied face - when it begins to emote and feel and possibly react to the viewer? Is it then more in the language of the animated character, or as we make it photo-realistic, the language of the video actor with deep dramatic back-story or simply as real as a person on the other side of the screen? A viewer cannot be rude to a portrait but can feel that they are being rude to an interactive character in an art installation. When does it become not an embodied face nor portrait but a mask - the icon that speaks of face but is never embodied? Masks also have a deep cultural, historic and ethnic language far different than that of human faces or art portraits. More eastern compared to the western portrait. Iconised faces such as the smiley face or the emoticon face, takes the mask full through to the western modern world of technology.

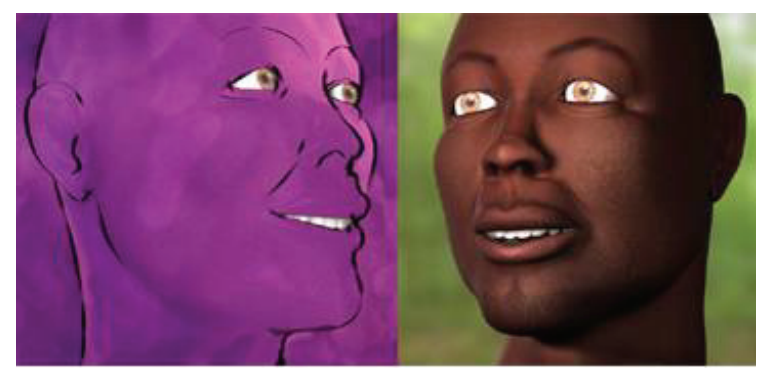

Figure 1: Same face model rendered 1) as a portrait and 2) realistically; stills from the autism research work

With animated and interactive art work, what is to stop a piece from fluidly moving between all these forms? This paper explores the synthetic face in art and science in all its forms and more importantly explores the virtuality of the face, for true art has one soul that can be manifest in many realisations and aesthetic communication forms. 
The paper will discuss where these forms differ and intersect both culturally and in relation to new technology (i.e. is the robot face more a face or more a mask, what about a game avatar). We then overview our computer facial application toolkit, which, using hierarchical parameterisation, can provide a comprehensive and open ended synthetic facial depiction, expression and communication system via: (1) the use of higherlevel parameters which apply lower level ones in combination and with constraints, and (2) defining time-based parameters that control facial actions. The system allows for experiments and questions that can be explored by traditional and new media artists, art historians, cognitive scientists and other scholars

\section{FACES AND PARAMETRIC FACE SPACE}

The face is one of the most important surfaces we deal with. It is the showcase of the self, displaying our sex, age, and ethnic background as well as our health and mood. It also is our main vehicle to transmit our feelings and explicit communications to others. We are all experts in interpreting, and communicating with the human face. We can recognise the face of someone we knew in the fifth grade, 20 years later. We can read volumes through one glance into our father's stare. Ironically, this universal expertise comes with little common understanding of what the mechanisms are that govern such talent (McNeil, 1998). Most people can't even describe the features of a loved one's face accurately nor can they dissect how it is that they know when someone is lying to them; they just know.

Visualising and, more recently, animating faces have long been a challenge to visual artists, animators, and communication professionals. The advent of computer-based tools opened a new chapter for this challenging task. Computergenerated face-based multimedia presentations play an increasing role in applications such as computer games, movies, communication, online services, as well as artistic endeavours. Delicate features and expressive capabilities make human faces both difficult and attractive to visualise and animate using computer based tools. There are many authoring tools for facial animation, but they are based on general modelling and animation techniques such as key framing, point morphing or boning systems. Very few can be used in an intuitive way specific to facial creation and understanding. A comprehensive and specially designed system for facial authoring can be used by expert and novice alike to explore how we use and perceive human facial communication, as well as to create and control synthetic faces for a myriad of industry, art and research applications. The primary requirement for developing such a system is to build in the knowledge space face modelling, both anatomically and behaviourally. We introduce FaceSpace as a basic face model in the form of a multidimensional parameter space that is a language for and controls facial geometry and behaviour. Our face-based systems, described later in the paper, are built on top of the FaceSpace model.

After earlier works by Parke (2000), many researchers have worked on different approaches to modelling the human face for creation and recognition purposes (Parke \& Waters, 2000). One common approach for facial animation is to model the underlying structure and interaction of bones, muscles and skin. This approach is based on the idea that simulating the underlying mechanisms of facial changes will lead to realistic visual results.

However, the complexity and subtlety of the human facial system along with the hyper-sensitivity of the viewer to the state of the face make this approach computationally prohibitive for interactive applications. Furthermore, a simulation approach is difficult to apply generally to a character with features that are impossible within real-world constraints, such as oblong eyes as well as other non-real or cartoon-like features. Another limitation of the simulation approach is that a particular expression is composed only of physical state (i.e. a set of tensed muscles, etc.) and is not linked to any semantic framework such as the associated emotional state of the character (e.g. sad, happy, quizzical, etc.)

\subsection{Parameter spaces}

Direct parameterisation models (Parke \& Waters, 2000) address the computational problems of simulation modelling by applying simple and efficient transformations of a head model through simple parameters such as eye-width, lip-thickness, or jaw-open. These parameters encapsulate highlevel knowledge about the combined effect of skin and muscle movement along with knowledge of the underlying mesh topology. They define the state of the head and act as 'dimensions' in a 'face space'.

We believe that direct parameterisation can provide a comprehensive and effective authoring system if extended through: (1) the use of higher-level pseudo-parameters which apply lower level parameters in combination and with constraints in order to produce complex expressions, and (2) defining time-based parameters that control actions and behaviours as an addition to the spatial geometry (for example parameters that control how expressions are performed or what typical head movements are to be created based on a personality type). 
Major contributions of this model are the inclusion of temporal and spatial parameters (e.g. expressions over time), intuitive parameter spaces (e.g. personality space), and hierarchical parameters with different levels of abstraction (e.g. heroicness built on top of simpler behaviour types). Besides the face-centric knowledge approach and weighing towards communication and behaviour of faces another goal of the FaceSpace model has been to decouple output details from the facecentric core, allowing for intuitive face oriented authoring which can be applied at any level, to any model, with any emotion. For instance -- add this expressive audio sequence to this cartoon face type but add more goofiness with a little heroicness to the personality as it animates through the given sequence outputting it as a 3D rendered movie; now take that same 'knowledge' sequence and try it on a realistic face with a more angry tone outputting it as image based movie. Also because the multidimensional parameters are align in a face centric way, it is also possible to affect a face from another knowledge or expression data stream, for instance one application of FaceSpace has been to remap emotional channels of music to emotional aspects of the face (DiPaola \& Arya, $2004 \& 2006$ ). Newer extensions to the parameter space approach include depiction controls which allow fine art painterly portrait depiction, which is aligned both with artistic painterly knowledge (the cognitive painterly process) as well as knowledge from vision and perception science (how humans see and perceive). In the following sections, we briefly review some related works, describe the basic concepts of FaceSpace, and introduce some FaceSpace-based systems.

The essence of the FaceSpace environment is a set of numerical parameters, each of which controls some aspect of a character's face, expression, emotion, movement and depiction. Parameters are typically unitised vectors, each representing a subroutine, which performs some low level complex transformations on the part of the face it controls. Because parameters are abstracted from their low level techniques, they have mathematically rigorous properties such as the ability to be combined, subtracted, added together, while still maintaining controllable and repeatable effects to their face model.

Parameters can be varied independently to modify specific features of the face (e.g. cheek-bone prominence, forehead height, jaw-width, etc.). This authoring paradigm is highly flexible, allowing a wide range of applications. The entire set of parameters can be exposed individually for full low level authoring control or a sub-set of these parameters with constraints can be presented to a novice user for customisation and personalisation. Higher-level constructs can be imposed on the basic parameter scheme by combining low-level parameters to create application-specific descriptive elements. For example a user could modify the character's appearance from 'sophisticated' to 'silly' with a single control that simultaneously modifies eye separation, forehead height, nose scale, etc.

Groups of high-level parameters can act on the face simultaneously, creating lip-sync speech with one channel while specifying an astonished look for the whole face on another independent channel. Because of their associative properties and their abstraction from the actual face topology, results typically always look natural, although naturalness can be arbitrary as you move away from realistic either towards fine art portrait depiction or cartoon exaggeration. In fact one of the driving forces behind our system is the ability to explore different dimensions of face spaces, to begin to understand faces as a language just like the language of cinema or painting or modern jazz. FaceSpace allows the concept of faces and face expressions to be explored at intuitive level.

\section{FACE SYSTEM EXAMPLES}

Once a believable, controllable and communicative face environment is available to artists, developers and researchers, we believe a new range of socialbased applications are possible. Most computer based communication systems such as internetbased websites (using HTML, PHP, ASP), information kiosks or ebooks (using PDF, Director, Flash) for example are informational in nature, not socially-based. People however (like teachers, aquarium or science experts, or museum guides) use a more social-based techniques to convey their message - they use their passion for the subject, narrative techniques, lesson plans, flexible content depending on audience or audience feedback, eye contact, humour, voice modulation. We believe these socially-based techniques using a communicative face system can open up more human centric applications in many areas, such as:

- Video Games that can convey the subtle dramatic nuances more common to cinema thereby extending games to a wider audience and into the educational and adult realms (Arya, DiPaola, and Parush, (2009).

- Chat Systems that use voice and facial expression for better and deeper communication.

- Education Systems that bring the passion of a teacher or expert scientist into distance education.

- Art and science research facial systems to better understand how humans perceive, receive and create social and emotional communication and expressions with faces. 
For our example sections, we will concentrate on applications that support more engaging art, learning and research facial systems and will discuss five ongoing application prototypes.

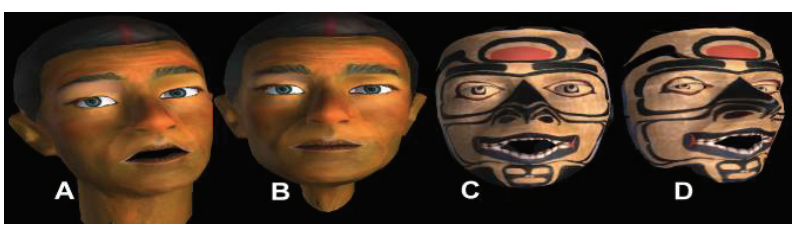

Figure 2: Frames from 'storytelling mask' interactive, where a real artist's voice, passion and expression first introduces himself $(A)$ transform into his artwork $(B, C)$ tells its back story with full voice and expression (D) and can return to his persona to interactively give interactive and educational content $(A)$

\subsection{Storytelling masks}

Most art or science museums (including zoos and aquariums) often still use static displays of text and graphics to explain the deeper historical or scientific concepts about the nearby artefact (i.e. a portrait, a model of a planet) and often the display is not read. The situation is very different when a human guide gives a presentation about that same artefact, engrossing the viewers in that subject as they use narrative, real-time and socially based deliveries. Can this experience be mimicked with interactive systems allowing students, who do not have geographically or financial access to a science facility a similar level of engagement and educational experience. Can a facility create a better level of engagement when a human guide is not available? We will describe two active prototypes:

Museums of anthropology, especially in North America, display a variety of artefacts from 'first nations' (Native Americans). Among the most attractive of these artefacts are masks and head figures presented on objects such as totem poles. Songs, myths, and stories relate these figures to the history of the people who made them. Computer-generated characters with those figureforms who also tell their stories and sing their songs are appealing and informative for the viewers and also provide a new means of creativity and expression to the native artists. Combination of FaceSpace design and scripting tools provide such a creative environment. In this specific case we have begun working with the Parks Department of British Columbia, Canada and the Native community to create a museum display where a virtual version of an artist appears and tells the story of his work, can virtually turn into the artwork - a native mask - and have a virtual version of the art tell its story (see Figure 2). Because all of this is under computer control it is possible to create many of the perceptual and educational techniques that a live human guide/artist could achieve including:

- Introduction: the ability to announce and bring the audience to the work;

- Narrative Style: conveying the back-story, passion, timing and expressiveness;

- Multiple Contexts: via interactive control, the material can be tailored to different age levels, different perspectives and focus areas, including updating the material;

- Presentation: the exhibit can feel more like a live presentation: for instance the interplay between artist and artefact - i.e. the mask is not displayed until the artist gives sufficient context; afterward the mask returns back to the artist/guide for additional commentary;

- Q\&A: at session end, the viewers can pick questions for more tailored commentary.

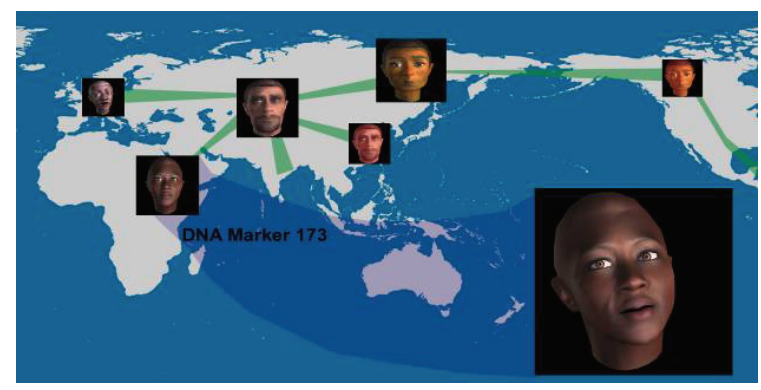

Figure 3: Screenshot of 'Evolving Faces' where emotive talking faces describe the DNA science of human migration out of Africa, actively 'morphing' facial types

\subsection{Evolving faces}

With similar goals and techniques of the Storytelling Mask project, Evolving Faces attempts to use facial agents to better engage viewers into the content, but in this case the agents are used to describe complicated scientific details, as well as create agents that are also an integral part of the content, evolving their appearance to tell the story of man's migration out of Africa based on new DNA techniques. Our FaceSpace systems allow a designer to create head models that correspond to various stages of human evolution, and assign different types of behaviour (e.g. coarse or fine) to them to be expressed during talking or interaction. Such characters are ideal for science booths or online learning. Adding simple or complicated artificial intelligence can improve the behavioural capability of the characters for real-time interaction. The display uses voices, change and expressive faces and maps rather than charts and text.

Screen shot from the Human Migration interactive is shown in Figure 3. It shows how complicated subject matter, how we migrated from Africa some 
$50-100,000$ years ago with its DNA marker and facial type migration evidence; can be engagingly put forth. Viewers can click on a specific face/area and have it tell the story of that DNA marker or click on a migratory path, having an evolving face explain the science facts of the journey of man. We have currently begun trials using a similar approach where marine scientist from different parts of the world could discuss their active work through facial systems next to marine displays in a major aquarium. The visitors would receive current (updated monthly) information from an expert, while the scientist would keep their privacy by just supply audio narrative to the education staff of the aquarium, who would put a face persona and behavioural state to their science narrative.

\subsection{MusicFace Application}

Music-driven Emotionally Expressive Face (MusicFace) (DiPaola \& Arya, 2004 \& 2006) is a multimedia application based on our facial systems to demonstrate the concept of Affective Communication Remapping, i.e. transforming emotional information from one communication medium to another. Emotional information is extracted from a piece of music by analysing musical features such as rhythm, energy, timbre, articulation and melody (Figure 4) which then automatically drives emotional face animation. Fine art versions of this work have been shown in galleries in NYC and Los Angles. We have been in discussion with a theatre owner to use the system to drive (computer projected) set design in real-time as it reacts to the music, much as a dancer might.

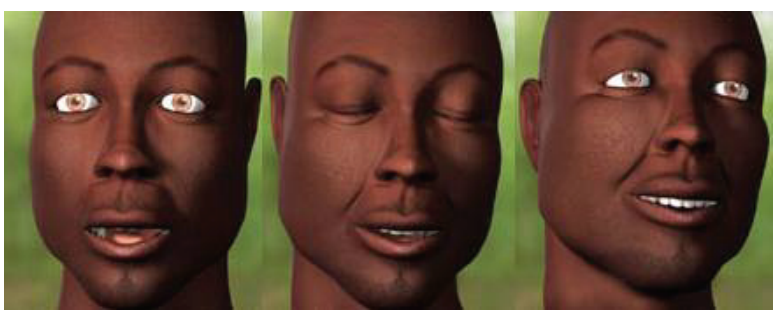

\begin{tabular}{|l|l|l|}
\hline Emotion & \multicolumn{1}{|c|}{ Feature } & \multicolumn{1}{c|}{ Value } \\
\hline Fear & $\begin{array}{l}\text { Tempo } \\
\text { Sound Level } \\
\text { Articulation }\end{array}$ & $\begin{array}{l}\text { Irregular } \\
\text { Low } \\
\text { Mostly non-legato }\end{array}$ \\
\hline Anger & $\begin{array}{l}\text { Tempo } \\
\text { Sound Level } \\
\text { Articulation }\end{array}$ & $\begin{array}{l}\text { Very rapid } \\
\text { Load } \\
\text { Mostly non-legato }\end{array}$ \\
\hline Happiness & $\begin{array}{l}\text { Tempo } \\
\text { Sound Level } \\
\text { Articulation }\end{array}$ & $\begin{array}{l}\text { Fast } \\
\text { Moderate or load } \\
\text { Airy }\end{array}$ \\
\hline
\end{tabular}

Figure 4: (a) Sample stills from MusicFace's music controlled animations and (b) Example of the parameters of music features and emotions that automatically drive the face animation

\subsection{Autism Communication Research}

Autism is one of the most commonly diagnosed developmental disorders in children. It is characterised by severe impairment in the domains of social, communicative, cognitive, and behavioural functioning. Despite the inconsistent profiles across individuals with autism, recent research reflects a developing consensus that the most central behavioural symptom of autism is the impairment of social behaviour, such as impairment of the social use of gaze, failure of joint attention interaction, lack of interest in peers, difficulty initiating communication, preference for solitary play. Autism affects from 5 to 17 per cent of the population.

Research has shown that individuals with autism fail to understand the emotional state expressed by another person (Joseph \& Tanaka, 2003). Whether individuals with autism rely on isolated parts of the face, such as eyes or bushy eyebrows to recognise faces rather than whole faces is not clear. That is because static images do not provide realistic assessment of face processing and emotion recognition.

Our research is in studying social competency and facial expression awareness in autistic children by combining our configurable 3D facial communication input system with a real-time eye gaze measurement system. This study involves varying the visual and social complexity of a dynamic 3D face in discrete steps to measure facial awareness and social competency (emotional recognition). The goal of the work is to both: 1) better understand this issue from a scientific and clinical point of view and 2) to build a computer based communication and serious game systems that take advantage of the clinical and technical results of the research.

Our study investigates whether different facial image rendering styles as well as varying levels of expressions scenarios, such as percent of direct eye gaze and normal to dampened mouth movement, can affect autistic children's visual perception and their social competence. We are using 'The Awareness of Social Inference Test' TASIT to measure results. The TASIT test comprises videoed sequences that can measure social and emotion recognition. By presenting each social sequence under controlled face animations with differing rendering styles and levels of expression situations on high developed autistic teenagers, we can 1) monitor eye-gaze patterns and retention time along with 2) how well they correctly identify the emotion associated with TASIT animated sequence. Currently most autism individuals miss important eye/eye brow expression cues by overly gazing at the quick movement 
mouth area. Our first set of experiments will systematically dampen mouth movements in perceptually valid ways, so the lip syncing will looking natural, but will less and less overwhelm the subject. We than can track at what level of mouth movement dampening, balancing eye gaze between eye, mouth and other head areas returns. Other experiments involve varying the percentage of direct eye gaze as well as changing the level of realism of rendering from very realistic to non photorealistic drawn like rendering. The goal is to understand how the complicated factors of social complexity versus visual complexity effect face to face communication in autism subjects. Because autism is a spectrum, individuals vary widely in how they perceive faces. Being able to deconstruct, output and modify expression units at any level of the animated 3D face animations allows us to conduct very detailed and modifiable experiments as well as easily transfer knowledge from the experiments over to consumer learning serious games toolkits for autism (see Figure 5).

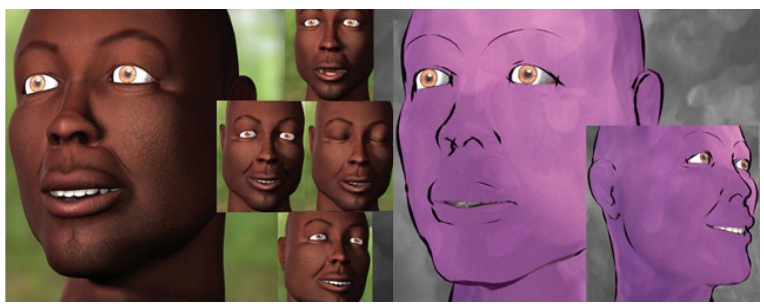

Figure 5: Stills from our facespace based TASIT social sequences using expression units $3 D$ rendered realistically (left) and in a drawing style (right)

\subsection{Art \& vision research: Rembrandt's genius}

Portrait artists and painters in general have over centuries developed a little understood, intuitive and open methodology that exploits cognitive mechanisms in the human perception and visual system. Compared with the original live sitter or the sitter's photograph, the portrait artist is able to filter and manipulate using what cognitive scientists only recently have begun to understand about our visual and perception system including shape and edge detection, centre of vision focusing and eye movement as well as colour temperature relational space. Our research, by first collecting (through interviews and reference data) a 'soft' qualitative knowledge space of how portrait painters achieve their craft, converts that soft data into a parameterised computer model which sits on the rigor of vision, image processing and facial knowledge. This computer model is the basis for our XML based interactive knowledge toolkit that has a twofold interdisciplinary goal. First it is able to generate a correlated space of painterly rendered portraits from input photographs in a large style set which has applications in computer based rendering, often called Non Photorealistic rendering
(NPR), for games and multimedia and second it can be used as a toolkit to explore interdisciplinary questions about the act of portrait painting by traditional and new media artists, art historians, cognitive scientists and other scholars. For the later area, it can begin to bridge these areas and show how artistic knowledge can lead to additional cognitive and human mind discoveries as well as understand a deeply creative, intuitive and human process of visual art making.

Our research system uses a parameterised approach to approximate a knowledge domain for painterly rendering of portraits. The knowledge domain uses fuzzy knowledge rules gained from interviews with oil portrait painters, data from the traditional 'portrait painter process' combined with human vision techniques and semantic models of the face and upper torso. By knowledge domain we mean that the system attempts to act on the same semantic level a human painter might function at, such as: human vision techniques, facial planes and expression, tonal masses, and colour temperature control. It also relies on an historical, open methodology that artists have created and passed down to each other. This qualitative knowledge is parameterised into an n-dimensional space of low level rules which can be accessed at different semantic levels. Non-professional photographic imagery of people's heads is used as input. Figure 1 shows the process starting at the top left, which can be summarised as collecting a knowledge space of the painterly process (qualitative at this point - lower left) from artists interviews, reference materials and user studies, then using known computer science and cognitive science models and methods, parameterise the knowledge (bottom middle) into the NPR system (lower left) which can be used for 1) better/wider NPR results as well as 2) clues/data into human vision/perception theory which can be mapped back into the system.

A painted portrait differs from a photo in that the artist intentionally selects only certain regions for fine detail (i.e., narrow versus broad brushwork) and the contrast level of edges. Although artists and art critics have claimed that these choices guide the viewer's eyes, this claim has not been thoroughly tested. In past studies of viewers gazing at original works of art, interpretation is complicated because regions of fine and coarse detail also differ in other ways (e.g., whether they are foreground or background). Here we monitored the gaze of participants viewing photos and paintings of the same portrait view, inspired by Rembrandt's portraits (e.g., Self Portrait with Beret, 1659). The paintings were created by a face space based nonphotorealistic rendering technique to mimic Rembrandt's style (DiPaola, 2009). In each painting, four regions of interest were selected for 
systematic variation in level of detail: left versus right eye region in finer detail and left versus right collar region in finer detail. Participants viewed each portrait for a 5 second period - in the context of viewing many portraits rendered in a wide range of styles, assigning ratings of artistic merit to each portrait. Analysis of the gaze patterns showed that fewer fixations were made overall when viewing paintings than photos, and that viewer's eyes were both attracted to and dwelt longer in the eye region of a portrait that was rendered in finer detail. Even regions of the paintings that were rarely fixated directly (i.e. collar regions below each face) nevertheless guided the gaze of viewers, specifically enhancing the salience of eyes rendered in fine detail on the same side of the portrait. This implies that Rembrandt and other master portraitists use an effective implicit theory of gaze direction (DiPaola et al., 2010).

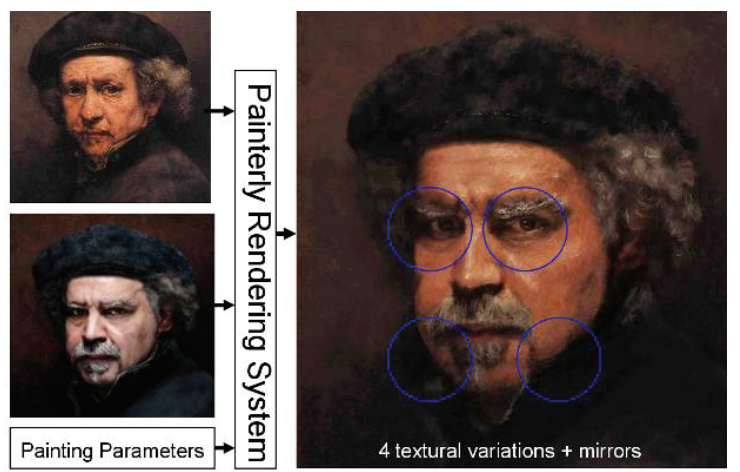

Figure 6: Using the painterly extension of our facespace system for scholarly analysis of Rembrandt to prove he intuited vision science, through painterly portrait simulation with eye tracking

\section{CONCLUSION}

It is well known that we humans have specific neuro-programming for recognising and interpreting faces. It is hoped that we can use the FaceSpace systems to better understand the conscious and intuitive meaning of faces and the universal language they appear to represent (facial meaning, inter-relationships and expressions) for use in the arts, cultural theory, science research and communication/expression systems. We hope that by creating a development kit that our team or other developers can use and customise, that it is possible to both gain a greater understanding of how we communicate and beguile using our faces as well as create an authoring environment that will let us take advantage of this new understanding via intuitive synthetic facial systems. In this paper, a modular multi-dimensional parameter space for face creation, animation and depiction is described as an underlying structure that will allow for this face-centric, knowledge-based, approach. We try to look at this parameter space not as a pure graphic model but an expressive and behavioural one for a 'communicative face'. The foundation of our model is a hierarchical geometry. Parameters are encapsulated within objects in a tree structure that allow local control of level-of-details both for animation and depiction.

The main advantages of our model are modular design, efficient and local control of geometric and depiction details, and inclusion of behavioural timebased extensions. Future work will focus on a more systematic parameterisation of facial emotions and personality in order to simplify navigation through the spatio-temporal face space. This modular face centric approach, we hope allows for more open ended research and exploration into the language of facial expression which hopefully can be used to both provide intrinsic knowledge to scholars and allows for creating new and innovative human centric expression systems.

\section{REFERENCES}

Arya, A., DiPaola, S. and Parush, A. (2009) Perceptually Valid Facial Expressions for Character-based Applications. International Journal of Computer Games Technology, vol 2009

McNeil, D. (1998) The Face. Little Brown and Company.

DiPaola, S. and Arya, A. (2004) Affective Communication Remapping in MusicFace System. Electronic Imaging \& Visual Arts, London.

DiPaola, S. and Arya, A. (2006) Emotional Remapping of Music to Facial Animation. In ACM Siggraph Video Game Symposium Proceedings.

DiPaola, S. (2009) Exploring a Parameterized Portrait Painting Space. International Journal of Art and Technology, 2, 1-2, pp. 82-93.

DiPaola S, Riebe C, Enns J, (2010) Rembrandt's Textural Agency: A Shared Perspective in Visual Art and Science", Leonardo, April, 43, 2.*

Joseph, R. and Tanaka, J. (2003) Holistic and partbased face recognition in children with autism. $J$ Child Psychol Psychiatry, May, 44, 4, pp. 529-42.

Parke, F. I. and Waters, K. (2000) Computer Facial Animation. A. K. Peters. 